\title{
Spaces of Ideal Convergent Sequences
}

\author{
M. Mursaleen ${ }^{1}$ and Sunil K. Sharma ${ }^{2}$ \\ ${ }^{1}$ Department of Mathematics, Aligarh Muslim University, Aligarh 202002, India \\ ${ }^{2}$ Department of Mathematics, Model Institute of Engineering \& Technology, Kot Bhalwal, J $~ K ~ 181122$, India
}

Correspondence should be addressed to M. Mursaleen; mursaleenm@gmail.com

Received 20 August 2013; Accepted 26 November 2013; Published 28 January 2014

Academic Editors: F. Başar and J. Xu

Copyright (c) 2014 M. Mursaleen and S. K. Sharma. This is an open access article distributed under the Creative Commons Attribution License, which permits unrestricted use, distribution, and reproduction in any medium, provided the original work is properly cited.

In the present paper, we introduce some sequence spaces using ideal convergence and Musielak-Orlicz function $\mathscr{M}=\left(M_{k}\right)$. We also examine some topological properties of the resulting sequence spaces.

\section{Introduction and Preliminaries}

The notion of ideal convergence was first introduced by Kostyrko et al. [1] as a generalization of statistical convergence which was further studied in topological spaces by Das et al. see [2]. More applications of ideals can be seen in $[2,3]$. We continue in this direction and introduce $I$-convergence of generalized sequences with respect to Musielak-Orlicz function.

A family $\mathscr{I} \subset 2^{X}$ of subsets of a nonempty set $X$ is said to be an ideal in $X$ if

(1) $\phi \in \mathscr{I}$,

(2) $A, B \in \mathscr{I}$ imply $A \cup B \in \mathscr{I}$,

(3) $A \in \mathscr{I}, B \subset A$ imply $B \in \mathscr{I}$,

while an admissible ideal $\mathscr{I}$ of $X$ further satisfies $\{x\} \in \mathscr{I}$ for each $x \in X$; see [1]. A sequence $\left(x_{n}\right)_{n \in \mathbb{N}}$ in $X$ is said to be $I$-convergent to $x \in X$. If for each $\epsilon>0$, the set $A(\epsilon)=\{n \epsilon$ $\mathbb{N}:\left\|x_{n}-x\right\| \geq \epsilon$ \} belongs to $\mathscr{F}$; see [1]. For more details about ideal convergent sequence spaces, see [4-10] and references therein.

Mursaleen and Noman [11] introduced the notion of $\lambda$ convergent and $\lambda$-bounded sequences as follows.

Let $\lambda=\left(\lambda_{k}\right)_{k=1}^{\infty}$ be a strictly increasing sequence of positive real numbers tending to infinity; that is,

$$
0<\lambda_{0}<\lambda_{1}<\cdots, \quad \lambda_{k} \longrightarrow \infty \quad \text { as } k \longrightarrow \infty .
$$

The sequence $x=\left(x_{k}\right) \in w$ is $\lambda$-convergent to the number $L$, called the $\lambda$-limit of $x$, if $\Lambda_{m}(x) \rightarrow L$, as $m \rightarrow \infty$, where

$$
\Lambda_{m}(x)=\frac{1}{\lambda_{m}} \sum_{k=1}^{m}\left(\lambda_{k}-\lambda_{k-1}\right) x_{k} .
$$

The sequence $x=\left(x_{k}\right) \in w$ is $\lambda$-bounded if $\sup _{m}\left|\Lambda_{m}(x)\right|<$ $\infty$. It is well known [11] that if $\lim _{m} x_{m}=a$ in the ordinary sense of convergence, then

$$
\lim _{m}\left(\frac{1}{\lambda_{m}}\left(\sum_{k=1}^{m}\left(\lambda_{k}-\lambda_{k-1}\right)\left|x_{k}-a\right|\right)\right)=0 .
$$

This implies that

$$
\lim _{m}\left|\Lambda_{m}(x)-a\right|=\lim _{m}\left|\frac{1}{\lambda_{m}} \sum_{k=1}^{m}\left(\lambda_{k}-\lambda_{k-1}\right)\left(x_{k}-a\right)\right|=0,
$$

which yields that $\lim _{m} \Lambda_{m}(x)=a$ and hence $x=\left(x_{k}\right) \in w$ is $\lambda$-convergent to $a$.

Let $X$ be a linear metric space. A function $p: X \rightarrow \mathbb{R}$ is called paranorm if

(1) $p(x) \geq 0$, for all $x \in X$,

(2) $p(-x)=p(x)$, for all $x \in X$,

(3) $p(x+y) \leq p(x)+p(y)$, for all $x, y \in X$, 
(4) if $\left(\lambda_{n}\right)$ is a sequence of scalars with $\lambda_{n} \rightarrow \lambda$ as $n \rightarrow$ $\infty$ and $\left(x_{n}\right)$ is a sequence of vectors with $p\left(x_{n}-x\right) \rightarrow$ 0 as $n \rightarrow \infty$, then $p\left(\lambda_{n} x_{n}-\lambda x\right) \rightarrow 0$ as $n \rightarrow \infty$.

A paranorm $p$ for which $p(x)=0$ implies that $x=0$ is called total paranorm and the pair $(X, p)$ is called a total paranormed space. It is well known that the metric of any linear metric space is given by some total paranorm (see [12, Theorem 10.4.2, P-183]). For more details about sequence spaces, see [13-15] and references therein.

An Orlicz function $M$ is a function which is continuous, nondecreasing, and convex with $M(0)=0, M(x)>0$ for $x>0$ and $M(x) \rightarrow \infty$ as $x \rightarrow \infty$.

Lindenstrauss and Tzafriri [16] used the idea of Orlicz function to define the following sequence space. Let $w$ be the space of all real or complex sequences $x=\left(x_{k}\right)$. Then,

$$
\ell_{M}=\left\{x \in w: \sum_{k=1}^{\infty} M\left(\frac{\left|x_{k}\right|}{\rho}\right)<\infty\right\}
$$

which is called an Orlicz sequence space. The space $\ell_{M}$ is a Banach space with the norm

$$
\|x\|=\inf \left\{\rho>0: \sum_{k=1}^{\infty} M\left(\frac{\left|x_{k}\right|}{\rho}\right) \leq 1\right\} .
$$

It is shown in [16] that every Orlicz sequence space $\ell_{M}$ contains a subspace isomorphic to $\ell_{p}(p \geq 1)$. The $\Delta_{2^{-}}$ condition is equivalent to $M(L x) \leq k L M(x)$ for all values of $x \geq 0$ and for $L>1$.

A sequence $\mathscr{M}=\left(M_{k}\right)$ of Orlicz function is called a Musielak-Orlicz function see; $[17,18]$. A sequence $\mathcal{N}=\left(N_{k}\right)$ defined by

$$
N_{k}(v)=\sup \left\{|v| u-\left(M_{k}\right): u \geq 0\right\}, \quad k=1,2, \ldots,
$$

is called the complementary function of a Musielak-Orlicz function $\mathscr{M}$. For a given Musielak-Orlicz function $\mathscr{M}$, the Musielak-Orlicz sequence space $t_{\mathscr{M}}$ and its subspace $h_{\mathscr{M}}$ are defined as follows:

$$
\begin{gathered}
t_{\mathscr{M}}=\left\{x \in w: I_{\mathscr{M}}(c x)<\infty \text { for some } c>0\right\}, \\
h_{\mathscr{M}}=\left\{x \in w: I_{\mathscr{M}}(c x)<\infty \text { for all } c>0\right\},
\end{gathered}
$$

where $I_{\mathscr{M}}$ is a convex modular defined by

$$
I_{\mathscr{M}}(x)=\sum_{k=1}^{\infty} M_{k}\left(x_{k}\right), \quad x=\left(x_{k}\right) \in t_{\mathscr{M}} .
$$

We consider $t_{\mathscr{M}}$ equipped with the Luxemburg norm

$$
\|x\|=\inf \left\{k>0: I_{\mathscr{M}}\left(\frac{x}{k}\right) \leq 1\right\} \text {, }
$$

or equipped with the Orlicz norm

$$
\|x\|^{0}=\inf \left\{\frac{1}{k}\left(1+I_{\mathscr{M}}(k x)\right): k>0\right\} .
$$

Let $\mathscr{M}=\left(M_{k}\right)$ be a Musielak-Orlicz function and let $p=\left(p_{k}\right)$ be a bounded sequence of positive real numbers. We define the following sequence spaces:

$$
\begin{aligned}
& c^{I}(\mathscr{M}, \Lambda, p) \\
& =\left\{x=\left(x_{k}\right) \in w: I-\lim _{k} M_{k}\left(\frac{\left|\Lambda_{k}(x)-L\right|}{\rho}\right)^{p_{k}}=0,\right. \\
& \text { for some } L \text { and } \rho>0\}, \\
& c_{0}^{I}(\mathscr{M}, \Lambda, p) \\
& \quad\left\{x=\left(x_{k}\right) \in w: I-\lim _{k} M_{k}\left(\frac{\left|\Lambda_{k}(x)\right|}{\rho}\right)^{p_{k}}=0,\right. \\
& \quad \text { for some } \rho>0\}, \quad\left\{x=\left(x_{k}\right) \in w: \sup _{k} M_{k}\left(\frac{\left|\Lambda_{k}(x)\right|}{\rho}\right)^{p_{k}}<\infty,\right. \\
& \left.l_{\infty}(\mathscr{M}, \Lambda, p)=\{x\} \text { for some } \rho>0\right\}
\end{aligned}
$$

We can write

$$
\begin{aligned}
& m^{I}(\mathscr{M}, \Lambda, p)=c^{I}(\mathscr{M}, \Lambda, p) \cap l_{\infty}(\mathscr{M}, \Lambda, p) \\
& m_{0}^{I}(\mathscr{M}, \Lambda, p)=c_{0}^{I}(\mathscr{M}, \Lambda, p) \cap l_{\infty}(\mathscr{M}, \Lambda, p) .
\end{aligned}
$$

If we take $p=\left(p_{k}\right)=1$, for all $k \in \mathbb{N}$, we have

$c^{I}(\mathscr{M}, \Lambda)$

$$
=\left\{x=\left(x_{k}\right) \in w: I-\lim _{k} M_{k}\left(\frac{\left|\Lambda_{k}(x)-L\right|}{\rho}\right)=0,\right.
$$

for some $L$ and $\rho>0\}$,

$$
c_{0}^{I}(\mathscr{M}, \Lambda)=\left\{x=\left(x_{k}\right) \in w: I-\lim _{k} M_{k}\left(\frac{\left|\Lambda_{k}(x)\right|}{\rho}\right)=0,\right.
$$
for some $\rho>0\}$,

$l_{\infty}(\mathscr{M}, \Lambda)=\left\{x=\left(x_{k}\right) \in w: \sup _{k} M_{k}\left(\frac{\left|\Lambda_{k}(x)\right|}{\rho}\right)<\infty\right.$, for some $\rho>0\}$. 
The following inequality will be used throughout the paper. If $0 \leq p_{k} \leq \sup p_{k}=H, D=\max \left(1,2^{H-1}\right)$, then

$$
\left|a_{k}+b_{k}\right|^{p_{k}} \leq D\left\{\left|a_{k}\right|^{p_{k}}+\left|B_{k}\right|^{p_{k}}\right\}
$$

for all $k$, and $a_{k}, b_{k} \in \mathbb{C}$. Also $|a|^{p_{k}} \leq \max \left(1,|a|^{H}\right)$ for all $a \in \mathbb{C}$.

The main aim of this paper is to study some ideal convergent sequence spaces defined by a Musielak-Orlicz function $\mathscr{M}=\left(M_{k}\right)$. We also make an effort to study some topological properties and prove some inclusion relations between these spaces.

\section{Main Results}

Theorem 1. Let $\mathscr{M}=\left(M_{k}\right)$ be a Musielak-Orlicz function and let $p=\left(p_{k}\right)$ be a bounded sequence of positive real numbers. Then, the spaces $c^{I}(\mathscr{M}, \Lambda, p), c_{0}^{I}(\mathscr{M}, \Lambda, p), m^{I}(\mathscr{M}, \Lambda, p)$, and $m_{0}^{I}(\mathscr{M}, \Lambda, p)$ are linear.

Proof. Let $x, y \in c^{I}(\mathscr{M}, \Lambda, p)$ and let $\alpha, \beta$ be scalars. Then, there exist positive numbers $\rho_{1}$ and $\rho_{2}$ such that

$$
\begin{aligned}
& I-\lim _{k} M_{k}\left(\frac{\left|\Lambda_{k}(x)-L_{1}\right|}{\rho_{1}}\right)^{p_{k}}=0, \quad \text { for some } L_{1} \in \mathbb{C}, \\
& I-\lim _{k} M_{k}\left(\frac{\left|\Lambda_{k}(y)-L_{2}\right|}{\rho_{2}}\right)^{p_{k}}=0, \quad \text { for some } L_{2} \in \mathbb{C} .
\end{aligned}
$$

For a given $\epsilon>0$, we have

$$
\begin{aligned}
& D_{1}=\left\{k \in \mathbb{N}: M_{k}\left(\frac{\left|\Lambda_{k}(x)-L_{1}\right|}{\rho_{1}}\right)^{p_{k}}\right\}, \\
& D_{2}=\left\{k \in \mathbb{N}: M_{k}\left(\frac{\left|\Lambda_{k}(y)-L_{2}\right|}{\rho_{2}}\right)^{p_{k}}\right\} .
\end{aligned}
$$

Let $\rho_{3}=\max \left\{2|\alpha| \rho_{1}, 2|\beta| \rho_{2}\right\}$. Since $\mathscr{M}=\left(M_{k}\right)$ is nondecreasing convex function, so by using inequality (15), we have

$$
\begin{aligned}
& \lim _{k} M_{k}\left(\frac{\left|\Lambda_{k}\left((\alpha x+\beta y)-\left(\alpha L_{1}+\beta L_{2}\right)\right)\right|}{\rho_{3}}\right)^{p_{k}} \\
& \leq \lim _{k} M_{k}\left(\frac{|\alpha|\left|\Lambda_{k}(x)-L_{1}\right|}{\rho_{3}}+\frac{|\beta|\left|\Lambda_{k}(y)-L_{2}\right|}{\rho_{3}}\right)^{p_{k}} \\
& \leq \lim _{k} M_{k}\left(\frac{\left|\Lambda_{k}(x)-L_{1}\right|}{\rho_{1}}\right)^{p_{k}} \\
& \quad+\lim _{k} M_{k}\left(\frac{\left|\Lambda_{k}(y)-L_{2}\right|}{\rho_{2}}\right)^{p_{k}} .
\end{aligned}
$$

Now, by (17), we have

$$
\begin{aligned}
& \left\{k \in \mathbb{N}: \lim _{k} M_{k}\left(\frac{\left|\Lambda_{k}\left((\alpha x+\beta y)-\left(\alpha L_{1}+\beta L_{2}\right)\right)\right|}{\rho_{3}}\right)^{p_{k}}\right. \\
& >\epsilon\} \subset D_{1} \cup D_{2} .
\end{aligned}
$$

Therefore, $\alpha x+\beta y \in c^{I}(\mathscr{M}, \Lambda, p)$. Hence $c^{I}(\mathscr{M}, \Lambda, p)$ is a linear space. Similarly, we can prove that $c_{0}^{I}(\mathscr{M}, \Lambda, p)$, $m^{I}(\mathscr{M}, \Lambda, p)$, and $m_{0}^{I}(\mathscr{M}, \Lambda, p)$ are linear spaces.

Theorem 2. Let $\mathscr{M}=\left(M_{k}\right)$ be a Musielak-Orlicz function. Then,

$$
c_{0}^{I}(\mathscr{M}, \Lambda, p) \subset c^{I}(\mathscr{M}, \Lambda, p) \subset l_{\infty}(\mathscr{M}, \Lambda, p) .
$$

Proof. Let $x \in c^{I}(\mathscr{M}, \Lambda, p)$. Then, there exist $L \in \mathbb{C}$ and $\rho>0$ such that

$$
I-\lim _{k} M_{k}\left(\frac{\left|\Lambda_{k}(x)-L\right|}{\rho}\right)^{p_{k}}=0 .
$$

We have

$M_{k}\left(\frac{\left|\Lambda_{k}(x)\right|}{2 \rho}\right)^{p_{k}} \leq \frac{1}{2} M_{k}\left(\frac{\left|\Lambda_{k}(x)-L\right|}{\rho}\right)^{p_{k}}+M_{k} \frac{1}{2}\left(\frac{|L|}{\rho}\right)^{p_{k}}$.

Taking supremum over $k$ on both sides, we get $x \in$ $l_{\infty}(\mathscr{M}, \Lambda, p)$. The inclusion $c_{0}^{I}(\mathscr{M}, \Lambda, p) \subset c^{I}(\mathscr{M}, \Lambda, p)$ is obvious. Thus,

$$
c_{0}^{I}(\mathscr{M}, \Lambda, p) \subset c^{I}(\mathscr{M}, \Lambda, p) \subset l_{\infty}(\mathscr{M}, \Lambda, p)
$$

This completes the proof of the theorem.

Theorem 3. Let $\mathscr{M}=\left(M_{k}\right)$ be a Musielak-Orlicz function and let $p=\left(p_{k}\right)$ be a bounded sequence of positive real numbers. Then, $l_{\infty}(\mathscr{M}, \Lambda, p)$ is a paranormed space with paranorm defined by

$$
g(x)=\inf \left\{\rho>0: \sup _{k} M_{k}\left(\frac{\left|\Lambda_{k}(x)\right|}{\rho}\right)^{p_{k}} \leq 1\right\} .
$$

Proof. It is clear that $g(x)=g(-x)$. Since $M_{k}(0)=0$, we get $g(0)=0$. Let us take $x, y \in l_{\infty}(\mathscr{M}, \Lambda, p)$. Let

$$
\begin{aligned}
& B(x)=\left\{\rho>0: \sup _{k} M_{k}\left(\frac{\left|\Lambda_{k}(x)\right|}{\rho}\right)^{p_{k}} \leq 1\right\}, \\
& B(y)=\left\{\rho>0: \sup _{k} M_{k}\left(\frac{\left|\Lambda_{k}(y)\right|}{\rho}\right)^{p_{k}} \leq 1\right\} .
\end{aligned}
$$


Let $\rho_{1} \in B(x)$ and $\rho_{2} \in B(y)$. If $\rho=\rho_{1}+\rho_{2}$, then we have

$$
\begin{aligned}
\sup _{k} & M_{k}\left(\frac{\left|\Lambda_{k}(x+y)\right|}{\rho}\right) \\
\leq & \left(\frac{\rho_{1}}{\rho_{1}+\rho_{2}}\right) \sup _{k} M_{k}\left(\frac{\left|\Lambda_{k}(x)\right|}{\rho_{1}}\right) \\
& +\left(\frac{\rho_{2}}{\rho_{1}+\rho_{2}}\right) \sup _{k} M_{k}\left(\frac{\left|\Lambda_{k}(y)\right|}{\rho_{2}}\right) .
\end{aligned}
$$

Thus, $\sup _{k} M_{k}\left(|\Lambda(x+y)| /\left(\rho_{1}+\rho_{2}\right)\right)^{p_{k}} \leq 1$ and

$$
\begin{aligned}
g(x+y) \leq & \inf \left\{\left(\rho_{1}+\rho_{2}\right)>0: \rho_{1} \in B(x), \rho_{2} \in B(y)\right\} \\
\leq & \inf \left\{\rho_{1}>0: \rho_{1} \in B(x)\right\} \\
& +\inf \left\{\rho_{2}>0: \rho_{2} \in B(y)\right\} \\
= & g(x)+g(y) .
\end{aligned}
$$

Let $\sigma^{s} \rightarrow \sigma$, where $\sigma, \sigma^{s} \in \mathbb{C}$ and let $g\left(x^{s}-x\right) \rightarrow 0$ as $s \rightarrow \infty$. We have to show that $g\left(\sigma^{s} x^{s}-\sigma x\right) \rightarrow 0$ as $s \rightarrow \infty$. Let

$$
\begin{gathered}
B\left(x^{s}\right)=\left\{\rho_{s}>0: \sup _{k} M_{k}\left(\frac{\left|\Lambda_{k}\left(x^{s}\right)\right|}{\rho_{s}}\right)^{p_{k}} \leq 1\right\}, \\
B\left(x^{s}-x\right)=\left\{\rho_{s}^{\prime}>0: \sup _{k} M_{k}\left(\frac{\left|\Lambda_{k}\left(x^{s}-x\right)\right|}{\rho_{s}^{\prime}}\right)^{p_{k}} \leq 1\right\} .
\end{gathered}
$$

If $\rho_{s} \in B\left(x^{s}\right)$ and $\rho_{s}^{\prime} \in B\left(x^{s}-x\right)$, then we observe that

$$
\begin{gathered}
M_{k}\left(\frac{\left|\Lambda_{k}\left(\sigma^{s} x^{s}-\sigma x\right)\right|}{\rho_{s}\left|\sigma^{s}-\sigma\right|+\rho_{s}^{\prime}|\sigma|}\right) \\
\leq M_{k}\left(\frac{\left|\Lambda_{k}\left(\sigma^{s} x^{s}-\sigma x^{s}\right)\right|}{\rho_{s}\left|\sigma^{s}-\sigma\right|+\rho_{s}^{\prime}|\sigma|}+\frac{\left|\left(\sigma x^{s}-\sigma x\right)\right|}{\rho_{s}\left|\sigma^{s}-\sigma\right|+\rho_{s}^{\prime}|\sigma|}\right) \\
\leq \frac{\left|\sigma^{s}-\sigma\right| \rho_{s}}{\rho_{s}\left|\sigma^{s}-\sigma\right|+\rho_{s}^{\prime}|\sigma|} M_{k}\left(\frac{\left(\left|\Lambda_{k}\left(x^{s}\right)\right|\right)}{\rho_{s}}\right) \\
\quad+\frac{|\sigma| \rho_{s}^{\prime}}{\rho_{s}\left|\sigma^{s}-\sigma\right|+\rho_{s}^{\prime}|\sigma|} M_{k}\left(\frac{\left|\Lambda_{k}\left(x^{s}-x\right)\right|}{\rho_{s}^{\prime}}\right) .
\end{gathered}
$$

From the above inequality, it follows that

$$
M_{k}\left(\frac{\left|\Lambda_{k}\left(\sigma^{s} x^{s}-\sigma x\right)\right|}{\rho_{s}\left|\sigma^{s}-\sigma\right|+\rho_{s}^{\prime}|\sigma|}\right)^{p_{k}} \leq 1
$$

and, consequently,

$$
\begin{aligned}
& g\left(\sigma^{s} x^{s}-\sigma x\right) \leq \inf \left\{\left(\rho_{s}\left|\sigma^{s}-\sigma\right|+\rho_{s}^{\prime}|\sigma|\right)>0:\right. \\
&\left.\rho_{s} \in B\left(x^{s}\right), \rho_{s}^{\prime} \in B\left(x^{s}-x\right)\right\} \\
& \leq\left(\left|\sigma^{s}-\sigma\right|\right)>0 \inf \left\{\rho>0: \rho_{s} \in B\left(x^{s}\right)\right\} \\
&+(|\sigma|)>0 \inf \left\{\left(\rho_{s}^{\prime}\right)^{p_{n} / H}: \rho_{s}^{\prime} \in B\left(x^{s}-x\right)\right\} \\
& \longrightarrow 0 \quad \text { as } s \longrightarrow \infty .
\end{aligned}
$$

This completes the proof.

Theorem 4. Let $\mathscr{M}^{\prime}=\left(M_{k}^{\prime}\right)$ and $\mathscr{M}^{\prime \prime}=\left(M_{k}^{\prime \prime}\right)$ be MusielakOrlicz functions that satisfy the $\Delta_{2}$-condition. Then,

(i) $Z\left(\mathscr{M}^{\prime \prime}, \Lambda, p\right) \subseteq Z\left(\mathscr{M}^{\prime} \circ \mathscr{M}^{\prime \prime}, \Lambda, p\right)$,

(ii) $Z\left(\mathscr{M}^{\prime}, \Lambda, p\right) \cap Z\left(\mathscr{M}^{\prime \prime}, \Lambda, p\right) \subseteq Z\left(\mathscr{M}^{\prime}+\mathscr{M}^{\prime \prime}, \Lambda, p\right)$ for $Z=c^{I}, c_{0}^{I}, m^{I}, m_{0}^{I}$.

Proof. (i) Let $x \in c_{0}^{I}\left(\mathscr{M}^{\prime \prime}, \Lambda, p\right)$. Then, there exists $\rho>0$ such that

$$
I-\lim _{k} \mathscr{M}_{k}^{\prime \prime}\left(\frac{\left|\Lambda_{k}(x)\right|}{\rho}\right)^{p_{k}}=0 .
$$

Let $\epsilon>0$ and choose $\delta$ with $0<\delta<1$ such that $M_{k}^{\prime}(t)<\epsilon$ for $0 \leq t \leq \delta$. Write $y_{k}=M_{k}^{\prime \prime}\left(\left|\Lambda_{k}(x)\right| / \rho\right)^{p_{k}}$ and consider

$$
\lim _{\substack{0 \leq y_{k}<\delta \\ k \in \mathbb{N}}} M_{k}^{\prime}\left(y_{k}\right)=\lim _{\substack{y_{k}<\delta \\ k \in \mathbb{N}}} M_{k}^{\prime}\left(y_{k}\right)+\lim _{\substack{y_{k}>\delta \\ k \in \mathbb{N}}} M_{k}^{\prime}\left(y_{k}\right) .
$$

Since $\mathscr{M}=\left(M_{k}\right)$ satisfies $\Delta_{2}$-condition, we have

$$
\lim _{\substack{y_{k} \leq \delta \\ k \in \mathbb{N}}} M_{k}^{\prime}\left(y_{k}\right) \leq M_{k}^{\prime}(2) \lim _{\substack{y_{k} \leq \delta \\ k \in \mathbb{N}}}\left(y_{k}\right) .
$$

For $y_{k}>\delta$, we have

$$
y_{k}<\frac{y_{k}}{\delta}<1+\frac{y_{k}}{\delta} .
$$

Since $\mathscr{M}^{\prime}=\left(M_{k}^{\prime}\right)$ is nondecreasing and convex, it follows that

$$
M_{k}^{\prime}\left(y_{k}\right)<M_{k}^{\prime}\left(1+\frac{y_{k}}{\delta}\right)<\frac{1}{2} M_{k}^{\prime}(2)+\frac{1}{2} \frac{M_{k}^{\prime}\left(2 y_{k}\right)}{\delta} .
$$

Since $\mathscr{M}^{\prime}=\left(M_{k}^{\prime}\right)$ satisfies $\Delta_{2}$-condition, we have

$$
M_{k}^{\prime}\left(y_{k}\right)<\frac{1}{2} K \frac{y_{k}}{\delta} M_{k}^{\prime}(2)+\frac{1}{2} K \frac{y_{k}}{\delta} M_{k}^{\prime}(2)=K \frac{y_{k}}{\delta} M_{k}^{\prime}(2) .
$$

Hence,

$$
\lim _{\substack{y_{k}>\delta \\ k \in \mathbb{N}}} M_{k}^{\prime}\left(y_{k}\right) \leq \max \left(1, K \delta^{-1} M_{k}^{\prime}(2)\right) \lim _{\substack{y_{k}<\delta \\ k \in \mathbb{N}}}\left(y_{k}\right) .
$$


From (32), (34), and (38), we have $x=\left(x_{k}\right) \in c_{0}^{I}\left(\mathscr{M}^{\prime}\right.$ 。 $\left.\mathscr{M}^{\prime \prime}, \Lambda, p\right)$. Thus, $c_{0}^{I}\left(\mathscr{M}^{\prime \prime}, \Lambda, p\right) \subseteq c_{0}^{I}\left(\mathscr{M}^{\prime} \circ \mathscr{M}^{\prime \prime}, \Lambda, p\right)$. Similarly, we can prove the other cases.

(ii) Let $x \in c_{0}^{I}\left(\mathscr{M}^{\prime}, \Lambda, p\right) \cap c_{0}^{I}\left(\mathscr{M}^{\prime \prime}, \Lambda, p\right)$. Then, there exists $\rho>0$ such that

$$
\begin{gathered}
I-\lim _{k} M_{k}^{\prime}\left(\frac{\left|\Lambda_{k}(x)\right|}{\rho}\right)^{p_{k}}=0, \\
I-\lim _{k} M_{k}^{\prime \prime}\left(\frac{\left|\Lambda_{k}(x)\right|}{\rho}\right)^{p_{k}}=0 .
\end{gathered}
$$

The rest of the proof follows from the following equality:

$$
\begin{aligned}
& \lim _{k \in \mathbb{N}}\left(M_{k}^{\prime}+M_{k}^{\prime \prime}\right)\left(\frac{\left|\Lambda_{k}(x)\right|}{\rho}\right)^{p_{k}} \\
& \quad=\lim _{k \in \mathbb{N}} M_{k}^{\prime}\left(\frac{\left|\Lambda_{k}(x)\right|}{\rho}\right)^{p_{k}}+\lim _{k \in \mathbb{N}} M_{k}^{\prime \prime}\left(\frac{\left|\Lambda_{k}(x)\right|}{\rho}\right)^{p_{k}} .
\end{aligned}
$$

Corollary 5. Let $\mathscr{M}=\left(M_{k}\right)$ be a Musielak-Orlicz function which satisfies $\Delta_{2}$-condition. Then, $Z(p, \Lambda) \subseteq Z(\mathscr{M}, \Lambda, p)$ holds for $Z=c^{I}, c_{0}^{I}, m^{I}$, and $m_{0}^{I}$.

Proof. The proof follows from Theorem 3 by putting $M_{k}^{\prime \prime}(x)=$ $x$ and $M_{k}^{\prime}(x)=M_{k}(x) \forall x \in[0, \infty)$.

Theorem 6. The spaces $c_{0}^{I}(\mathscr{M}, \Lambda, p)$ and $m_{0}^{I}(\mathscr{M}, \Lambda, p)$ are solid.

Proof. We will prove for the space $c_{0}^{I}(\mathscr{M}, \Lambda, p \Lambda)$. Let $x \in$ $c_{0}^{I}(\mathscr{M}, \Lambda, p)$. Then, there exists $\rho>0$ such that

$$
I-\lim _{k} M_{k}\left(\frac{\left|\Lambda_{k}(x)\right|}{\rho}\right)^{p_{k}}=0 .
$$

Let $\left(\alpha_{k}\right)$ be a sequence of scalars with $\left|\alpha_{k}\right| \leq 1 \forall k \in \mathbb{N}$. Then, the result follows from the following inequality:

$$
\lim _{k} M_{k}\left(\frac{\left|\Lambda_{k}(\alpha x)\right|}{\rho}\right)^{p_{k}} \leq \lim _{k} M_{k}\left(\frac{\left|\Lambda_{k}(x)\right|}{\rho}\right)^{p_{k}}
$$

and this completes the proof. Similarly, we can prove for the space $m_{0}^{I}(\mathscr{M}, \Lambda, p)$.

Corollary 7. The spaces $c_{0}^{I}(\mathscr{M}, \Lambda, p)$ and $m_{0}^{I}(\mathscr{M}, \Lambda, p)$ are monotone.

Proof. It is easy to prove, so we omit the details.

Theorem 8. The spaces $c^{I}(\mathscr{M}, \Lambda, p)$ and $c_{0}^{I}(\mathscr{M}, \Lambda, p)$ are sequence algebra.
Proof. Let $x, y \in c_{0}^{I}(\mathscr{M}, \Lambda, p)$. Then,

$$
\begin{gathered}
I-\lim _{k} M_{k}\left(\frac{\left|\Lambda_{k}(x)\right|}{\rho_{1}}\right)^{p_{k}}=0, \quad \text { for some } \rho_{1}>0, \\
I-\lim _{k} M_{k}\left(\frac{\left|\Lambda_{k}(y)\right|}{\rho_{2}}\right)^{p_{k}}=0, \quad \text { for some } \rho_{2}>0 .
\end{gathered}
$$

Let $\rho=\rho_{1}+\rho_{2}$. Then, we can show that

$$
I-\lim _{k} M_{k}\left(\frac{\left|\Lambda_{k}(x \cdot y)\right|}{\rho}\right)^{p_{k}}=0 .
$$

Thus, $(x \cdot y) \in c_{0}^{I}(\mathscr{M}, \Lambda, p)$. Hence, $c_{0}^{I}(\mathscr{M}, \Lambda, p)$ is a sequence algebra. Similarly, we can prove that $c^{I}(\mathscr{M}, \Lambda, p)$ is a sequence algebra.

\section{Conflict of Interests}

The authors declare that there is no conflict of interests regarding the publication of this paper.

\section{References}

[1] P. Kostyrko, T. Šalát, and W. Wilczyński, "I-convergence," Real Analysis Exchange, vol. 26, no. 2, pp. 669-685, 2000.

[2] P. Das, P. Kostyrko, W. Wilczyński, and P. Malik, "I and $I^{*}$ convergence of double sequences," Mathematica Slovaca, vol. 58, no. 5, pp. 605-620, 2008.

[3] P. Das and P. Malik, "On the statistical and $I$ variation of double sequences," Real Analysis Exchange, vol. 33, no. 2, pp. 351-363, 2008.

[4] V. Kumar, "On $I$ and $I^{*}$-convergence of double sequences," Mathematical Communications, vol. 12, no. 2, pp. 171-181, 2007.

[5] M. Mursaleen and A. Alotaibi, "On I-convergence in random 2-normed spaces," Mathematica Slovaca, vol. 61, no. 6, pp. 933940, 2011.

[6] M. Mursaleen, S. A. Mohiuddine, and O. H. H. Edely, "On the ideal convergence of double sequences in intuitionistic fuzzy normed spaces," Computers \& Mathematics with Applications, vol. 59, no. 2, pp. 603-611, 2010.

[7] M. Mursaleen and S. A. Mohiuddine, "On ideal convergence of double sequences in probabilistic normed spaces," Mathematical Reports, vol. 12(62), no. 4, pp. 359-371, 2010.

[8] M. Mursaleen and S. A. Mohiuddine, "On ideal convergence in probabilistic normed spaces," Mathematica Slovaca, vol. 62, no. 1, pp. 49-62, 2012.

[9] A. Şahiner, M. Gürdal, S. Saltan, and H. Gunawan, "Ideal convergence in 2-normed spaces," Taiwanese Journal of Mathematics, vol. 11, no. 5, pp. 1477-1484, 2007.

[10] B. C. Tripathy and B. Hazarika, "Some $I$-convergent sequence spaces defined by Orlicz functions," Acta Mathematicae Applicatae Sinica, vol. 27, no. 1, pp. 149-154, 2011.

[11] M. Mursaleen and A. K. Noman, "On some new sequence spaces of non-absolute type related to the spaces $\ell_{p}$ and $\ell_{\infty} \mathrm{I}$," Filomat, vol. 25, no. 2, pp. 33-51, 2011.

[12] A. Wilansky, Summability through Functional Analysis, vol. 85 of North-Holland Mathematics Studies, North-Holland Publishing, Amsterdam, The Netherlands, 1984. 
[13] K. Raj and S. K. Sharma, "Some sequence spaces in 2-normed spaces defined by Musielak-Orlicz function," Acta Universitatis Sapientiae, vol. 3, no. 1, pp. 97-109, 2011.

[14] K. Raj and S. K. Sharma, "Some generalized difference double sequence spaces defined by a sequence of Orlicz-functions," Cubo, vol. 14, no. 3, pp. 167-190, 2012.

[15] K. Raj and S. K. Sharma, "Some multiplier sequence spaces defined by a Musielak-Orlicz function in $n$-normed spaces," New Zealand Journal of Mathematics, vol. 42, pp. 45-56, 2012.

[16] J. Lindenstrauss and L. Tzafriri, "On Orlicz sequence spaces," Israel Journal of Mathematics, vol. 10, pp. 379-390, 1971.

[17] L. Maligranda, Orlicz Spaces and Interpolation, vol. 5 of Seminars in Mathematics, Polish Academy of Science, 1989.

[18] J. Musielak, Orlicz Spaces and Modular Spaces, vol. 1034 of Lecture Notes in Mathematics, Springer, 1983. 


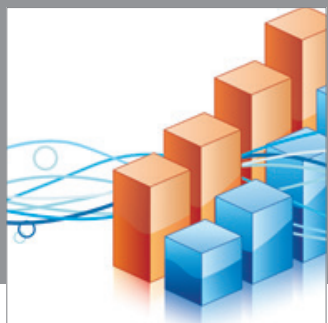

Advances in

Operations Research

mansans

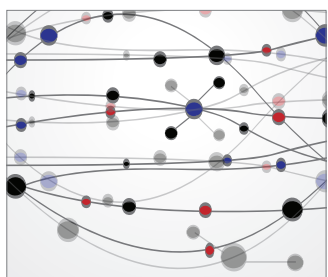

The Scientific World Journal
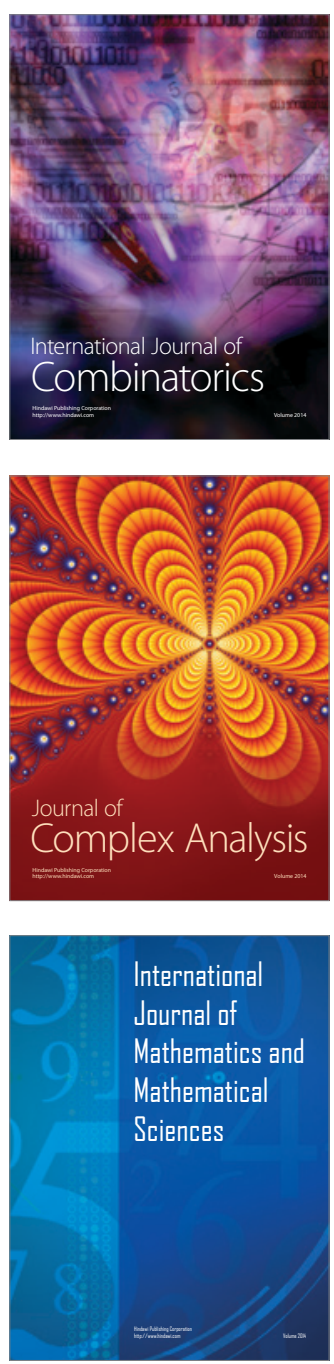
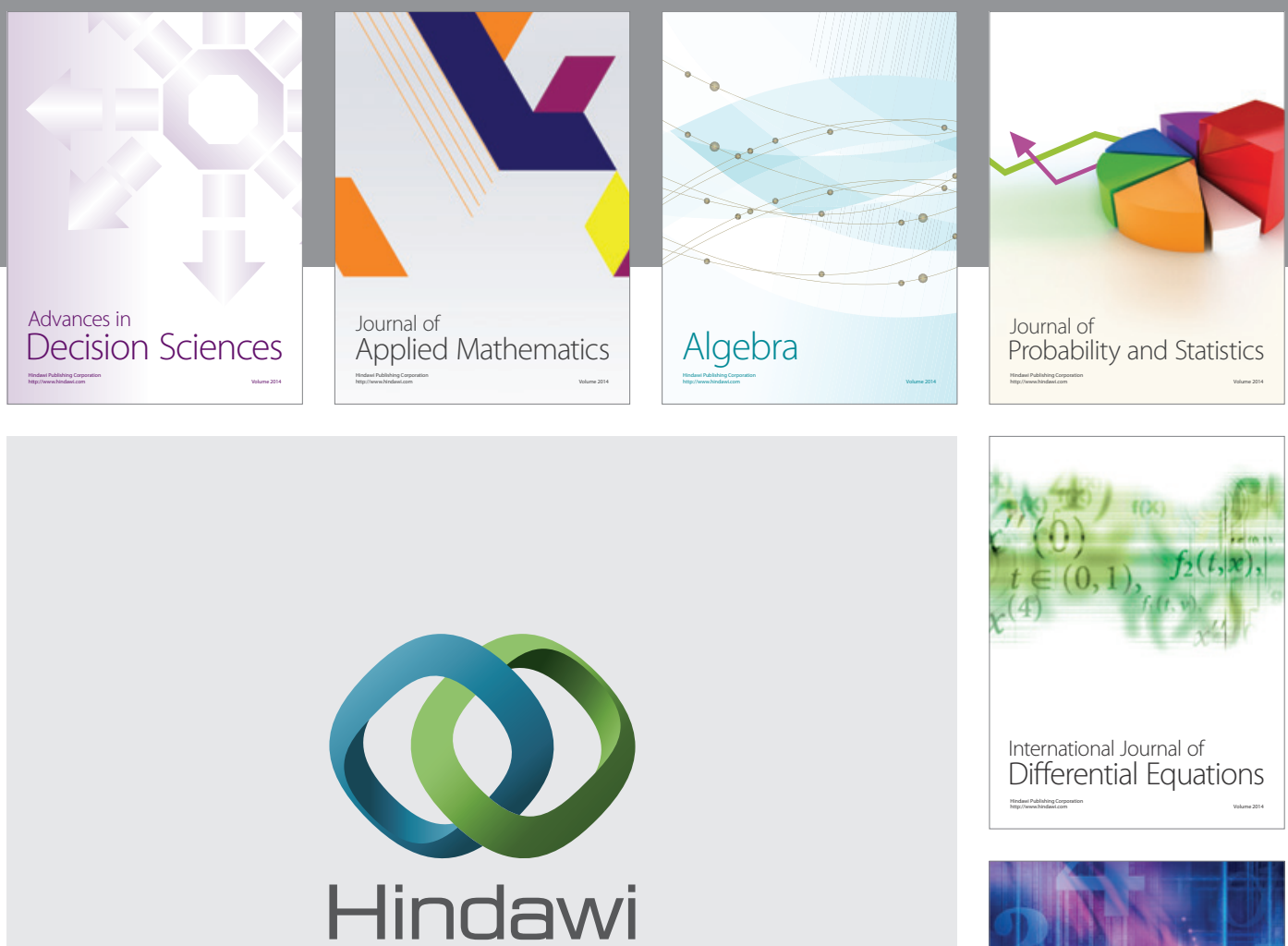

Submit your manuscripts at http://www.hindawi.com
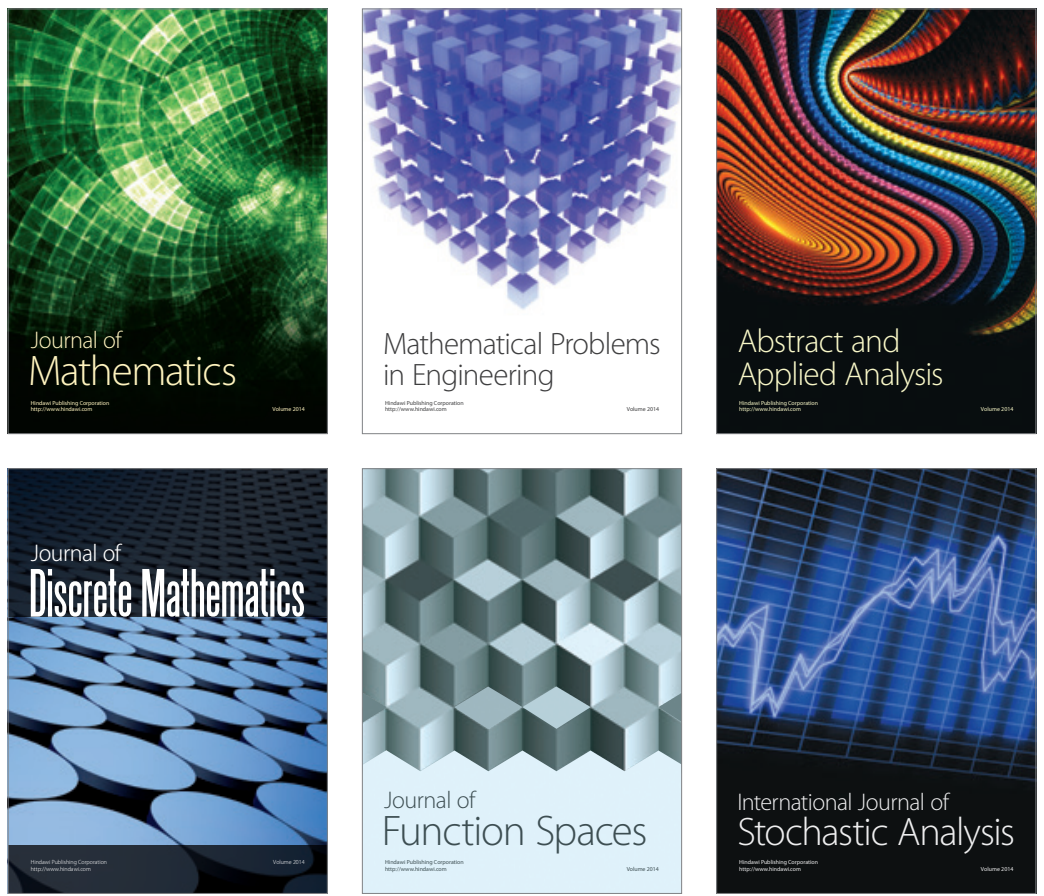

Journal of

Function Spaces

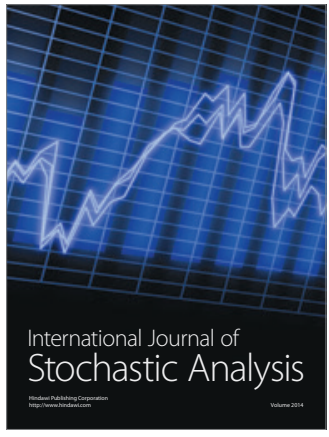

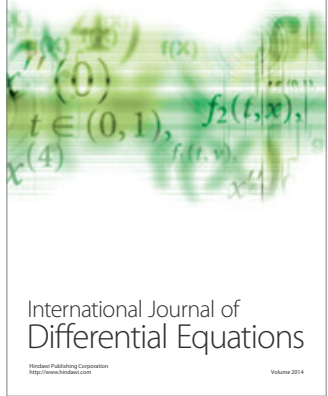
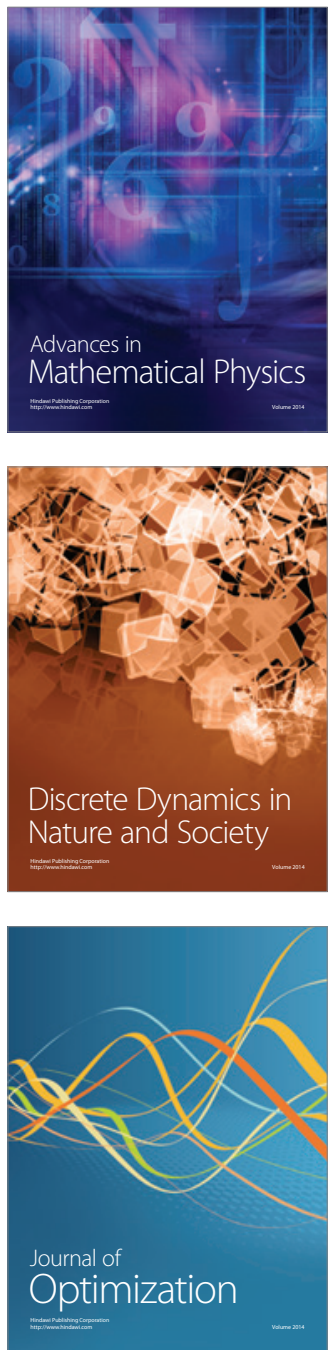